\title{
Morbidity in an Infant by Superabsorbent Polymer Ingestion
}

\author{
Henry Tan Chor Lip, ${ }^{1,2,3 *}$ Tan Jih Huei, ${ }^{1,2}$ V Muthualhagi A/P Vellusamy ${ }^{3}$ \\ 1 Department of General Surgery, Faculty of Medicine, National University of Malaysia Medical Centre, Kuala Lumpur, Malaysia \\ 2 Clinical Research Centre, Hospital Sultan Ismail, Johor Bahru, Malaysia \\ 3 Department of Pediatric Surgery, Hospital Sultanah Aminah, Johor Bahru, Malaysia
}

\section{ABSTRACT}

Superabsorbent polymer (SAP) ingestion is a potentially dangerous condition and may lead to morbidity and mortality. A 22-month-old girl presented with intestinal obstruction. At operation a SAP was found as a cause of intestinal obstruction. The child underwent second surgery for anastomotic leak and ileostomy was made.

\section{Key words: Superabsorbent polymer; Intestinal obstruction; Intestinal perforation; Morbidity; Foreign body ingestion}

Correspondence*: Henry Tan Chor Lip, Department of Paediatric Surgery, Hospital Sultanah Aminah, Jalan Abu Bakar, Masjid Sultan Abu Bakar, 80000 Johor Bahru, Johor, Malaysia.

E-mail: relos1402@gmail.com

Submitted: $25-07-2017$

Conflict of Interest: None
(C) 2017, Tan Chor Lip et al.

Accepted: 04-09-2017

Source of Support: Nil

This is an open-access article distributed under the terms of the Creative Commons Attribution License, which permits unrestricted use, distribution, and reproduction in any medium, provided the original work is properly cited.

\section{INTRODUCTION}

Polyacrylmide, also known as SAP, absorbs water and expands.[1] On ingestion, the size of polymer increases as it passes through the gastrointestinal tract. This can result in intestinal obstruction and even perforation.[1-3] Herein, we report a case of intestinal obstruction caused by ingestion of SAP.

\section{CASE REPORT}

A 22-month-old girl presented with abdominal distension and vomiting for the last 4 days. There were no signs of peritonitis. $X$ ray abdomen revealed dilated small bowel (Fig.1A). Child was resuscitated with IV fluids. Due to the lack of clinical improvement and increasing bilious aspirate, laparotomy was performed. A superabsorbent polymer ball was found lodged in the ileum approximately $30 \mathrm{~cm}$ proximal to the ileocaecal valve leading to intestinal obstruction. Small bowel proximal to the site of obstruction was distended, edematous and congested
(Fig.1B). An enterotomy with primary repair was performed after removal of the polymer (Fig.1C).
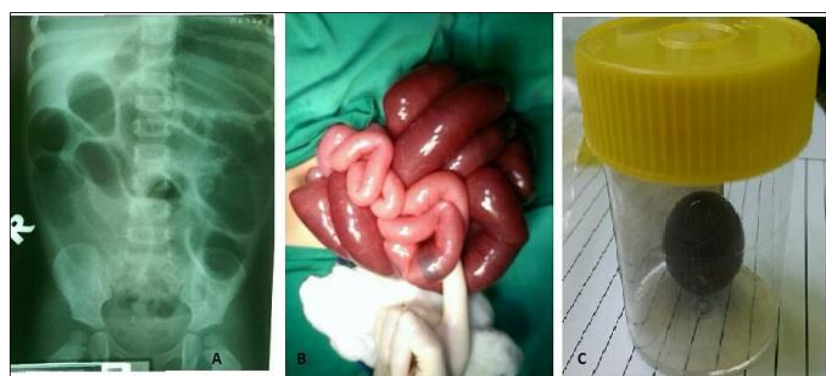

Figure 1: Abdominal radiograph supine view showing gas filled distended loops. No foreign body seen. B) Intra-operative findings of dilated, congested small intestines and lodged SAP as pointed out. C) SAP ball specimen after retrieval.

Patient was again explored on 6th postoperative day due to anastomotic leak. A temporary ileostomy was made. Post-operative recovery was uneventful and a reversal of the ileostomy was performed six days after emergency re-laparotomy. Recovery after reversal of 
ileostomy was uneventful and patient was discharged in a stable condition.

\section{DISCUSSION}

Most of the patients with SAP ingestion are infants. Diagnosis of a intraluminal rounded cystic structure has been reported on ultrasonography, CT scan and MRI abdomen.[1-8] SAP ball impaction usually reported in the small intestine. A mortality has been reported in literature due to SAP ingestion.[2] Since 2013, toys containing SAP are banned in Malaysia.

In our patient, the anastomotic leak was the main cause of morbidity. Another case of anastomotic dehiscence after primary closure of enterotomy after SAP retrieval has been reported in literature.[2] An in-vitro study which reenacts the same conditions after ingestion of SAP into the small intestines in a lab found that SAPs tend to expand maximally to almost 5 times its original diameter at 96 hours post ingestion.[3] In view of the expanding properties of SAP, expectant management is dangerous and if a positive history of SAP ingestion is present, an immediate endoscopic retrieval is recommended.[2,3].

Consent: Authors have submitted signed consent form from legal guardians of the patient for use of clinical material in this manuscript. The Consent form is available with Editorial office.
Authors' Contribution: All authors contributed equally in concept, literature review, and drafting of the manuscript and approved the final version of this manuscript.

\section{REFERENCES}

1. Mirza B, ljaz L, Sheikh A. Decorative crystal balls causing intestinal perforation. J Indian Assoc Pediatr Surg. 2011; 16:106-7.

2. Mirza B, Sheikh A. Mortality in a case of crystal gel ball ingestion: An alert for parents. APSP J Case Rep. 2012; 3:6.

3. Zamora IJ, Vui TL, Larimer LE, Olutoye O. Water-absorbing balls: A "growing" problem. Pediatrics. 2012; 130:1011-4.

4. Al-Saied G, Al-Malki T, Ayoub M, Hussain T, Thabet R, Gamal A, et al. Unusual cause of small bowel obstructions in infants: A warning letter to the parents. J Pediatr Surg Case Rep. 2016; 11:39-43.

5. Muthukumaran J, Vivek S. Intestinal obstruction due to accidental ingestion of hygroscopic foreign body. Indian Pediatr. 2014; 51:1022-3.

6. Moon JS, Bliss D, Hunter CJ. An unusual case of small bowel obstruction in a child caused by ingestion of waterstoring gel beads. J Pediatr Surg. 2012; 45:e19-e22.

7. Faizah MZ, Soon YY, Che DD, Yusof A, Dayang AA. Ultrasound diagnosis of crystal jelly ball ingestion causing intestinal obstruction. Austin J Radiol. 2016; 3:1046.

8. Pratap A, Mathur V, Tanger R, Gupta A, Kumar A. Foreign body in duodenum, mimicking a duplication cyst on imaging. APSP J Case Rep. 2016; 7:35. 